\title{
Approach for uncertain multi-attribute decision-making with interval numbers complementary preference relation on alternatives under partial weight information
}

\author{
Zhou Hong-an \\ School of Science, Xi'an Technological University, Xi'an, 710032, China \\ email: honganzhou@sohu.com
}

\begin{abstract}
Keywords: Uncertain Multi-attribute decision-making; Goal programming; Possibility; Priority
\end{abstract}
\begin{abstract}
The uncertain multi-attribute decision making (UMADM) problem that the information about attribute weights is known partly and the decision maker (DM) has preference information on alternatives in the form of interval numbers complementary judgment matrix is investigated. Firstly, based on interval numbers complementary matrix the objective decision information is uniformed by using a translation function and then a goal programming model is established. The attribute weights are obtained by solving the model and thus the overall values of every alternative are gained. Secondly, by using an existing priority formula of possibility the alternatives are ranked. An approach based on the goal programming model and possibility is proposed. It is characterized by simple operation and easy to implement on computer. Finally, a practical example is illustrated to show the feasibility and availability of the developed method.
\end{abstract}

\section{Introduction}

Because of the complexity of objective thing and the uncertainty of DM's understandings, moreover, the decision maker take part in the process of decision-making, that is the subjective preference information of DM is often used to rank alternatives, this makes the UMADM problems with uncertain preference information on alternatives become a hot topic of research in Refs [1-7]. At present, these problems have been investigated and some methods of solving them have been presented by some scholar. The UMADM problems that the information about attribute weights is unknown completely and the DM has interval numbers utility preference on alternatives are studied in Refs [2-3], the linear programming and least deviation method are proposed respectively. The UMADM problems that the attribute weights are interval numbers and DM's preference relation on alternatives is in the interval numbers reciprocal or complementary judgment matrix judgment matrix are investigated in Refs [4-5], the attribute weights are obtained by establishing a linear programming model, an approach based on interval positive ideal point of alternatives and projection to rank alternatives is presented in Ref [4]. The method based on based on the additive consistency of interval numbers complementary judgment matrix and C-OWA operator is presented in Ref [5]. With respect to UMADM problems that decision-making information are interval numbers and preference information on alternatives, the method of grey relational analysis is proposed in Ref [6]. The UMADM problems is investigated, in which the DM has preference information on alternatives in the form of interval reciprocal judgment matrix and the information about attribute weights take the form of interval complementary judgment matrix, a maximizing model based on the consistency of preference information is established to asses the weights of attributes in Ref [7]. But the interval attribute vales are made exact numbers, so there exist the loss of decision making information. Based on the above references a new approach will be proposed to solve the UMADM problems that the information about attribute weights is known partly and DM's preference information on alternatives take the form of interval numbers complementary judgment matrix in this paper. Firstly, we makes the objective decision information uniform based on interval numbers complementary matrix by using a translation function and then a goal programming model is established. The attribute weights are obtained by solving the model and thus the overall values of every alternative are gained. Secondly, by using an existing priority formula of possibility the 
alternatives are ranked. An approach based on the goal programming model and possibility is proposed. It is characterized by simple operation and easy to implement on computer. Finally, a practical example is illustrated to show the feasibility and availability of the developed method.

\section{Preliminary knowledge}

Definition 1 Let $\tilde{a}=\left[a^{L}, a^{R}\right]=\left\{x \mid 0 \leq a^{L} \leq x \leq a^{R}\right\}$, then $\tilde{a}$ is called an interval number. Specially, $\tilde{a}$ is degenerated to be a real number if $a^{L}=a^{R}$ in Ref [2].

In order to compare interval numbers, the following concept is introduced.

Definition 2 Let $\tilde{a}=\left[a^{L}, a^{R}\right], \quad \tilde{b}=\left[b^{L}, b^{R}\right]$, Then $p(\tilde{a} \geq \tilde{b})$ is called possibility of $\tilde{a} \geq \tilde{b}$ in Ref

[2], where $p(\tilde{a} \geq \tilde{b})=\min \left\{\max \left[\frac{a^{R}-b^{L}}{\left(a^{R}-a^{L}\right)+\left(b^{R}-b^{L}\right)}, 0\right], 1\right\}$

The basic model of the UMADM problem which will be studied in this paper can be represented as follows:

- Let $X=\left\{x_{1}, x_{2}, \cdots, x_{n}\right\}$ be a set of $n(\geq 2)$ feasible alternatives, and denote $N=\{1,2, \cdots, n\}$;

- Let $S=\left\{s_{1}, s_{2}, \cdots, s_{m}\right\}$ be a set of $m(\geq 2)$ attributes, and denote $M=\{1,2, \cdots, m\}$;

- Let $\omega=\left(\omega_{1}, \omega_{2}, \cdots, \omega_{m}\right)^{T}$ be the vector of weights, such that $\omega_{k}^{R} \geq \omega_{k} \geq \omega_{k}^{L} \geq 0$, $\sum_{k=1}^{m} \omega_{k}^{L} \leq 1, \sum_{k=1}^{m} \omega_{k}^{R} \geq 1, \sum_{k=1}^{m} \omega_{k}=1$, where $\omega_{k}^{L}$ and $\omega_{k}^{R}$ are the left and right bounds of $\omega_{k}$, respectively. And $\omega_{k}$ is the weight of attribute $s_{k}$;

- Let $\widetilde{A}=\left(\tilde{a}_{i j}\right)_{m \times n}$ be an objective decision matrix, where $\tilde{a}_{i j}=\left[a_{i j}^{L}, a_{i j}^{R}\right]$ is the attribute value for the alternative $x_{j}$ with respect to attribute $s_{i}, i \in M, j \in N$;

- Let $\tilde{B}=\left(\tilde{b}_{l j}\right)_{n \times n}$ be the DM's uncertain complementary preference matrix on alternatives, where $\tilde{b}_{l j}$ denotes a ratio of preference degree for the alternative $x_{l}$ over $x_{j}$, and such that $\tilde{b}_{l j}=\left[b_{l j}^{L}, b_{l j}^{R}\right], b_{l j}^{L}+b_{j l}^{R}=b_{l j}^{R}+b_{j l}^{L}=1, l, j \in N$.

In the general case, there is benefit and cost attribute values in the UMADM, and the different attribute values may be the different dimension. For the convenience of decision-making, we need to deal with all attributes in dimensionless units and normalize each attribute value. This can be achieved by normalizing $\tilde{a}_{i j}$ in the matrix $\widetilde{A}=\left(\tilde{a}_{i j}\right)_{m \times n}$ into a corresponding element $\tilde{r}_{i j}$ in the matrix $\widetilde{R}=\left(\tilde{r}_{i j}\right)_{m \times n}$ with the following formulas in Ref [2], where $\tilde{r}_{i j}=\left[r_{i j}^{L}, r_{i j}^{R}\right], i \in M, j \in N$.

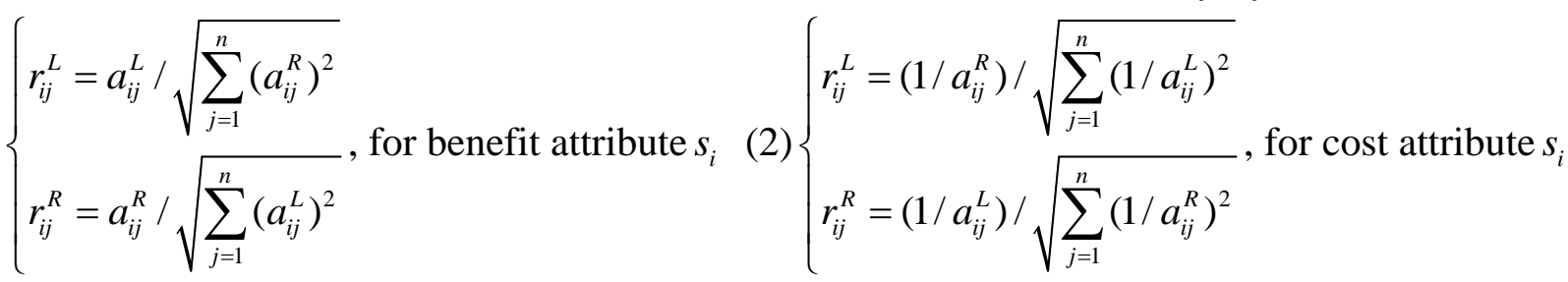

Using the simple additive weighting method, the overall value of alternative $x_{j}$ can be expressed as $\quad \tilde{z}_{j}(\omega)=\sum_{i=1}^{m} \omega_{i} \tilde{r}_{i j}, j \in N$

\section{Model and approach of decision-making}

Because the attribute weight $\omega$ is known partly, we must first determine it so that the alternatives are ranked. Considering that the overall value $\tilde{z}_{j}(\omega)$ is derived from the objective 
decision matrix $\widetilde{R}=\left(\tilde{r}_{i j}\right)_{m \times n}$, thus we here regard it as the objective preference values. In order to make the objective decision-making information and the DM's subjective preference on alternatives uniform, by using the operational laws of interval numbers in ref [2], we can transform $\tilde{z}_{j}(\omega)$ into uncertain complementary preference matrix $\tilde{D}=\left(\tilde{d}_{l j}\right)_{n \times n}, l, j \in N$, where $\tilde{d}_{l j}$ can be expressed as follows:

$$
\tilde{d}_{i j}=\frac{\sum_{k=1}^{m} \omega_{k} \tilde{r}_{k i}}{\sum_{k=1}^{m} \omega_{k}\left(\tilde{r}_{k i}+\tilde{r}_{k j}\right)}=\frac{\left[\sum_{k=1}^{m} \omega_{k} r_{k i}^{L}, \sum_{k=1}^{m} \omega_{k} r_{k i}^{R}\right]}{\left[\sum_{k=1}^{m} \omega_{k}\left(r_{k i}^{L}+r_{k j}^{L}\right), \sum_{k=1}^{m} \omega_{k}\left(r_{k i}^{R}+r_{k j}^{R}\right)\right]}=\left[\frac{\sum_{k=1}^{m} \omega_{k} r_{k i}^{L}}{\sum_{k=1}^{m} \omega_{k}\left(r_{k i}^{R}+r_{k j}^{R}\right)}, \frac{\sum_{k=1}^{m} \omega_{k} r_{k i}^{R}}{\sum_{k=1}^{m} \omega_{k}\left(r_{k i}^{L}+r_{k j}^{L}\right)}\right], i, j \in N .
$$

Obviously, the meaning of $\tilde{d}_{l j}$ in the matrix $\tilde{D}=\left(\tilde{d}_{l j}\right)_{n \times n}$ is similar to that of $\tilde{b}_{l j}$ in the matrix $\tilde{B}=\left(\tilde{b}_{l j}\right)_{n \times n}$. If the uncertain complementary preference relations $\tilde{D}$ and $\tilde{B}$ are consistent, then $\tilde{D}=\tilde{B}$, i.e., $\tilde{d}_{l j}=\tilde{b}_{l j}, l, j \in N$, and then we have

$$
\left\{\begin{array} { l } 
{ b _ { i j } ^ { L } = \sum _ { k = 1 } ^ { m } \omega _ { k } r _ { k i } ^ { L } / \sum _ { k = 1 } ^ { m } \omega _ { k } ( r _ { k i } ^ { R } + r _ { k j } ^ { R } ) } \\
{ b _ { i j } ^ { R } = \sum _ { k = 1 } ^ { m } \omega _ { k } r _ { k i } ^ { R } / \sum _ { k = 1 } ^ { m } \omega _ { k } ( r _ { k i } ^ { L } + r _ { k j } ^ { L } ) }
\end{array} \Leftrightarrow \left\{\begin{array}{l}
\sum_{k=1}^{m}\left(b_{i j}^{L} r_{k i}^{R}+b_{i j}^{L} r_{k j}^{R}-r_{k i}^{L}\right) \omega_{k}=0 \\
\sum_{k=1}^{m}\left(b_{i j}^{R} r_{k i}^{L}+b_{i j}^{R} r_{k j}^{L}-r_{k i}^{R}\right) \omega_{k}=0
\end{array}\right.\right.
$$

However, there always exist some deviations between the subjective preference values and the corresponding objective preference values on alternatives in the real life. This is, (5) often does not hold. Hence, we introduce the deviation items $f_{l j}^{L}$ and $f_{l j}^{R}$, i.e. let

$$
f_{i j}^{L}=\left|\sum_{k=1}^{m}\left(b_{i j}^{L} r_{k i}^{R}+b_{i j}^{L} r_{k j}^{R}-r_{k i}^{L}\right) \omega_{k}\right| ; f_{i j}^{R}=\left|\sum_{k=1}^{m}\left(b_{i j}^{R} r_{k i}^{L}+b_{i j}^{R} r_{k j}^{L}-r_{k i}^{R}\right) \omega_{k}\right| .
$$

It is easy to know we can minimize all the deviation to determine the reasonable vector of attribute weights $\omega=\left(\omega_{1}, \omega_{2}, \cdots, \omega_{m}\right)^{T}$. Therefore, we can establish the following multi-objective optimization model:

$$
\begin{aligned}
& \min f_{i j}^{L}=\left|\sum_{k=1}^{m}\left(b_{i j}^{L} r_{k i}^{R}+b_{i j}^{L} r_{k j}^{R}-r_{k i}^{L}\right) \omega_{k}\right| ; \\
& \min f_{i j}^{R}=\left|\sum_{k=1}^{m}\left(b_{i j}^{R} r_{k i}^{L}+b_{i j}^{R} r_{k j}^{L}-r_{k i}^{R}\right) \omega_{k}\right| ; \\
& \text { s.t. } \omega_{i}^{R} \geq \omega_{i} \geq \omega_{i}^{L} \geq 0, \sum_{i=1}^{m} \omega_{i}^{L} \leq 1, \sum_{i=1}^{m} \omega_{i}^{R} \geq 1, \sum_{i=1}^{m} \omega_{i}=1, l, j \in N .
\end{aligned}
$$

Since all the above objective function are expected to be equal to zero, and considering all above object functions are fair, we can transform the model (M1) into the following goal programming model (M2):

(M2) $\min J=\sum_{i=1}^{n} \sum_{j=1}^{n}\left[\left(d_{i j}^{L+}+d_{i j}^{L-}\right)+\left(d_{i j}^{R+}+d_{i j}^{R-}\right)\right]$;

s.t. $\sum_{k=1}^{m}\left(b_{i j}^{L} r_{k i}^{R}+b_{i j}^{L} r_{k j}^{R}-r_{k i}^{L}\right) \omega_{k}-d_{i j}^{L+}+d_{i j}^{L-}=0 ; \sum_{k=1}^{m}\left(b_{i j}^{R} r_{k i}^{L}+b_{i j}^{R} r_{k j}^{L}-r_{k i}^{R}\right) \omega_{k}-d_{i j}^{R+}+d_{i j}^{R-}=0$;

$d_{i j}^{L-} d_{i j}^{L+}=0, d_{i j}^{R-} d_{i j}^{R+}=0 ; d_{i j}^{L-} \geq 0, d_{i j}^{L+} \geq 0, d_{i j}^{R-} \geq 0, d_{i j}^{R+} \geq 0 ;$ 
$\omega_{i}^{R} \geq \omega_{i} \geq \omega_{i}^{L} \geq 0, \sum_{i=1}^{m} \omega_{i}^{L} \leq 1, \sum_{i=1}^{m} \omega_{i}^{R} \geq 1, \sum_{i=1}^{m} \omega_{i}=1, i \in M, l, j \in N$

where $d_{l j}^{L+}$ and $d_{l j}^{L-}$ denote the upper and lower deviation variables of $\sum_{k=1}^{m}\left(b_{i j}^{L} r_{k i}^{R}+b_{i j}^{L} r_{k j}^{R}-r_{k i}^{L}\right) \omega_{k}$ whose expected value is equal to zero, respectively; $d_{l j}^{R+}$ and $d_{l j}^{R-}$ denote the upper and lower deviation variables of $\sum_{k=1}^{m}\left(b_{i j}^{R} r_{k i}^{L}+b_{i j}^{R} r_{k j}^{L}-r_{k i}^{R}\right) \omega_{k}$ whose expected value is equal to zero, respectively.

By using LIGO software the model (M2) is solved and the vector of attribute weights $\omega$ can be obtained. Substituting $\omega$ into (6), we can get the overall value $\tilde{z}_{j}(\omega)$ of the alternative $x_{j}$.

Because $\tilde{z}_{j}(\omega)(j \in N)$ are an interval numbers. In order to obtain the priority of alternative, we can calculate the possibility $p\left(\tilde{z}_{i} \geq \tilde{z}_{j}\right) \triangleq p_{i j}$ of $\tilde{z}_{i} \geq \tilde{z}_{j}$ by using (1) and establish the possibility matrix $P=\left(p_{i j}\right)_{n \times n}$, it is easy to know $P=\left(p_{i j}\right)_{n \times n}$ is a numerical complementary judgment matrix. Let $v=\left(v_{1}, v_{2}, \cdots, v_{n}\right)^{T}$ is the ranking vector of $P=\left(p_{i j}\right)_{n \times n}$, then

$$
v_{i}=\frac{\sum_{j=1}^{n} p_{i j}+\frac{n}{2}-1}{n(n-1)}, i \in N
$$

Obviously, the bigger the vales of $v_{i}$ is, the better the corresponding alternative $x_{j}$.

Based on the above discussion, we give the solving processes and show the feasibility and availability of the proposed method by a practical example in the next section.

\section{Illustrative example}

An investment company is planning to exploit a new product and there are four investment alternatives $x_{j}(j=1,2,3,4)$ to be considered. The main attributes of evaluating the alternatives are listed as $s_{1}$ : investment amount, $s_{2}$ : expected net profit amount, $s_{3}$ : venture profit amount; $s_{4}$ : venture loss amount, respectively. Obviously, $s_{2}$ and $s_{3}$ are of benefit attributes, $s_{1}$ and $s_{4}$ are of cost attributes. Both the attribute values of every alternative and the weighted information $\omega_{i}(i=1,2,3,4)$ are listed in Table 1 (attribute unit is10000\$):

Table1 Decision matrix $\tilde{A}$ and weighted information

\begin{tabular}{|l|c|c|c|c|c|}
\hline$s_{i}$ & $\omega_{i}$ & $x_{1}$ & $x_{2}$ & $x_{3}$ & $x_{4}$ \\
\hline$s_{1}$ & {$[0.25,0.45]$} & {$[5,7]$} & {$[10,11]$} & {$[5,6]$} & {$[9,11]$} \\
\hline$s_{2}$ & {$[0.12,0.15]$} & {$[4,5]$} & {$[6,7]$} & {$[4,5]$} & {$[5,6]$} \\
\hline$s_{3}$ & {$[0.24,0.35]$} & {$[4,6]$} & {$[5,6]$} & {$[3,4]$} & {$[5,7]$} \\
\hline$s_{4}$ & {$[0.18,0.36]$} & {$[0.4,0.6]$} & {$[1.5,2]$} & {$[0.4,0.7]$} & {$[1.3,1.5]$} \\
\hline
\end{tabular}

Step1 By (2) and (3), we can get the normalized decision matrix $\tilde{R}$ from Table1as follows: 


$$
\widetilde{R}=\left(\widetilde{r_{i j}}\right)_{4 \times 4}=\left[\begin{array}{llll}
{[0.40,0.71]} & {[0.25,0.35]} & {[0.46 .0 .71]} & {[0.25,0.39]} \\
{[0.32,0.50]} & {[0.47,0.69]} & {[0.32,0.50]} & {[0.40,0.59]} \\
{[0.32,0.65]} & {[0.40,0.65]} & {[0.24,0.44]} & {[0.40,0.76]} \\
{[0.43,0.98]} & {[0.13,0.26]} & {[0.37,0.98]} & {[0.17,0.30]}
\end{array}\right]
$$

Step2 Suppose that the DM gives his/her uncertain complementary preference matrix $\tilde{B}=\left(\tilde{b}_{l j}\right)_{4 \times 4}$ on alternatives as follows:

$$
\tilde{B}=\left[\begin{array}{llll}
{[0.5,0.5]} & {[0.4,0.6]} & {[0.1,0.7]} & {[0.2,0.6]} \\
{[0.4,0.6]} & {[0.5,0.5]} & {[0.3,0.4]} & {[0.5,0.8]} \\
{[0.3,0.9]} & {[0.6,0.7]} & {[0.5,0.5]} & {[0.1,0.4]} \\
{[0.4,0.8]} & {[0.2,0.5]} & {[0.6,0.9]} & {[0.5,0.5]}
\end{array}\right]
$$

Then, by solving the model (M2), the weight vector of the attributes is obtained as $\omega=(0.32,0.15,0.35,0.18)^{T}$.

Step3 By (4), the overall value of each alternative are calculated and listed as follows: $\tilde{z}_{1}=[0.3654,0.7061] ; \tilde{z}_{2}=[0.3139,0.4898] ; \tilde{z}_{3}=[0.3458,0.6326] ; \tilde{z}_{4}=[0.3106,0.5333]$.

Step4 by (1) calculating possibility matrix as follows:

$$
P=\left[\begin{array}{cccc}
0.5 & 0.7592 & 0.5742 & 0.7020 \\
0.2408 & 0.5 & 0.3112 & 0.4496 \\
0.4258 & 0.6888 & 0.5 & 0.6320 \\
0.2980 & 0.5504 & 0.3680 & 0.5
\end{array}\right]
$$

Setp5 Using (6) to be obtained: $v_{1}=0.2946 ; \quad v_{2}=0.2085 ; \quad v_{3}=0.2706 ; v_{4}=0.2237$. So the priority of the corresponding alternative $x_{j}$ is $x_{2} \prec x_{4} \prec x_{3} \prec x_{1}$, That is, the best alternative is $x_{1}$.

\section{Conclusion}

In this paper, we propose a new method to solve the UMADM problems that the information about weights is known partly and the DM has uncertain complementary preference relation on alternatives. The core of this method is to determine the attribute weights based on the multiplicative consistency of interval numbers complementary judgment matrix. The proposed method can sufficiently utilize the objective information of alternatives and meet the subjective requirements of the DM as much as possible, and it is also characterized by simple operation and easy to implement on a computer. The numerical results have also indicated that the method is feasible and efficient.

\section{Acknowledgement}

In this paper, the research was sponsored by the Natural Science Research Foundation of Shaanxi Province (2015JQ1007)

\section{References}

[1] Hwang C L, Yoon K. Multiple Attribute Decision Making [M]. Berlin: Spring -Verlag, 1981.

[2] XU Ze-shui. Uncertain Multiple Attribute Decision Making: Methods and Applications. Beijing: Tsinghua University Publication House, 2004.

[3] JIANG Yan-ping, FAN Zhi-ping. Method for multiple attribute decision making with attribute interval numbers and preference information on alternatives. Journal of Systems Engineering and 
Electronics, 2005, 27 (2):250- 252.

[4] Xu Z S. On Method for Uncertain Multiple Attribute Decision Making Problems with Uncertain Multiplicative Preference Information on Alternatives. Fuzzy Optimization and Decision Making, 2005,4(2), 131-139.

[5] Zhou Hong-an, Liu San-yang, Fang Xiang-rong. Method for uncertain multi-attribute decision making with preference information in the form of interval numbers complementary judgment matrix [J]. Journal of Systems Engineering and Electronics,2007,18(2):265-269.

[6] WEI Gui-wu, WEI Yu. Model of Grey Relational Analysis for Interval Multiple Attribute Decision Making with Preference Information on Alternatives [J]. Chinese Journal of Management Science,,2008,16(1): 255-261.

[7]FENG Xiang-qian, WEI Cui-pin, QIAN Xiao-hua. An Approach to Multiple Attribute Decision Making based on the Consistency of Preference Information [J]. OPERATIONS RESEARCH AND MANAGEMENT SCIENCE, 2010, 19(4):73-77. 